\title{
Progesterone regimen impact on live birth rate and neonatal outcomes in artificial frozen-thawed embryo transfer cycles: a retrospective cohort study
}

\section{Yuan Liu}

Shanghai General Hospital, Shanghai Jiaotong University School of Medicine

Yu Wu ( $D$ wuyu1970@yahoo.com)

Shanghai General Hospital, Shanghai Jiaotong University School of Medicine https://orcid.org/00000001-7808-8725

\section{Research}

Keywords: FET, HRT, progesterone, live birth, birthweight

Posted Date: February 18th, 2020

DOI: https://doi.org/10.21203/rs.2.23836/v1

License: (c) (1) This work is licensed under a Creative Commons Attribution 4.0 International License. Read Full License 


\section{Abstract}

Background: Previous studies have demonstrated that singletons from frozen embryo transfer (FET) are heavier and longer-gestational-days at delivery than those from fresh embryo transfer. The amounts and routes of progesterone used in FET vary tremendously among different ART centers. Does different serum progesterone level induced by different progesterone regimens determine live birth rate and neonatal outcomes in hormone replacement therapy frozen-thawed embryo transfer (HRT-FET) cycles?

Design: A cohort study of 856 HRT-FET cycles from a Chinese public fertility center. Data from patients undergoing their first FET cycles from 2015-2018 were extracted from the database. All patients had their first FET with two day2 or day3 embryos transferred. Endometrial preparation was performed with sequential administration of estrogen followed by progesterone $60 \mathrm{mg}$ per day intramuscularly or Crinone $90 \mathrm{mg}$ per day vaginally. Live birth was the primary outcome. Secondary outcome included clinical pregnancy rate, singleton birthweight, large for gestational age (LGA) rate, SGA rate and preterm delivery rate. Student's t test, Mann-Whitney U-test, Chi square analysis and multivariable logistic regression were used where appropriate. Differences were considered significant if $p<0.05$.

Results: No significant difference of live birth rate was found between different progesterone regimens (Adjusted OR 1.128, 95\% Cl 0.842, 1.511, p=0.420). Neonatal outcomes like birthweight, preterm delivery rate, SGA and LGA rate were not different between two progesterone regimens. Serum $\mathrm{P}$ level $>41.82$ $\mathrm{pmol} / \mathrm{L}$ at 14 day post-FET was associated with higher live birth rate than serum $\mathrm{P}$ level $\leq 41.82 \mathrm{pmolL}$ in HRT-FET cycles when progesterone was intramuscularly delivered (Adjusted OR 1.690, 95\% Cl 1.002, $2.849, p=0.049$ ). Birthweight and gestational weeks were not different between these two different $\mathrm{P}$ level groups.

Conclusions: Progesterone vaginally or intramuscularly didn't impact live birth rate and neonatal outcomes in artificial FET cycles. Relatively higher serum progesterone level induced by intramuscular regimen did not increase newborn birthweight or prolong gestational weeks compared to vaginal regimen. Intramuscular progesterone supplementation during HRT-FET cycles was associated with improved live birth rate when progesterone concentration at day 14 post-FET was higher than $41.82 \mathrm{pmol} / \mathrm{L}$.

\section{Background}

As cryopreservation has been advanced to be an efficient and reliable laboratory procedure, freeze-all policy and elective embryo cryopreservation have been increasingly prevalent with a variety of clinic indications. FET has been welcomed as global trend in recent years due to multiple reasons including the endometrial synchrony and relative steady endocrine environment compared to supraphysiologic estrogen milieu generated by controlled ovarian stimulation (COS). However, we must recognize the adverse perinatal outcomes of FET, like higher risk of macrosomia, perinatal mortality and pregnancy complications like pre-eclampsia[1-4]. More reports suggested that singletons from FET were associated with higher risk of large and post-date babies and placenta accrete, compared to fresh transfer and natural pregnancy $[5,6]$. The reason behind that varied. Some reports suggested embryo cryopreservation 
altered epigenetics regulation inducing abnormal placentation and fetal growth[6]. More studies advised that the elevated level of estrogen's impact on endometrium made an essential role[7, 8].

The difference of gestational weeks and birthweight of singletons between FET and fresh IVF cycles led us to speculate the association between the quality of placentation and cycle characteristics. Though some evidence revealed that excessive estrogen priming set off the obstetrics complications, scarce literature has been focused on the supraphysiologic progesterone exposure to the deep placentation. Progestin has been suggested to directly advance vascular proliferation during placentation[9]. A supraphysiologic progestin exposure in HRT could initiate excessively deep placentation which would make a difference in infant birthweight and obstetrical consequences[10]. Since the regimen and amount of progesterone applied in HRT-FET cycles varied a lot from different IVF centers, there is little agreement on the ideal route of administration and dose. Considering the exogenous progesterone administered usually exceeds the endogenous progesterone of menstrual cycles by folds, our study aimed to explore whether circulating progesterone values induced by different progesterone regimen impact live birth rate, and more importantly the neonatal outcomes.

\section{Methods}

Study design and patients

The retrospective study was undertaken at the assisted reproduction medicine department in Shanghai general hospital affiliated to Shanghai Jiao Tong university school of medicine, including 856 women who had undergone their first FET from January 2015 to December 2018. Inclusion criteria were maternal age $<48$, undergoing two Day 2 or Day 3 cleavage-stage embryos transfer following HRT endometrium preparation. The patients with cryopreserved oocytes or donor oocytes and with prior attempts at conception via IVF and FET were excluded from the study. The final database included 856 women and 240 live birth singletons in the criteria. We keep following up by phone calls until one month after the patients' due date. Institutional review board and ethics committee of Shanghai General Hospital approval was obtained.

\section{IVF and Laboratory protocols}

Ovarian stimulation, oocyte retrieval and IVF/ICSI procedures have been previously described[11]. For IVF, oocytes were inseminated with human tubal fluid supplemented with $10 \%$ serum substitute supplement and with around 300000 progressively motile spermatozoa. For ICSI, oocytes were placed in the fertilization medium immediately after microinjection. Fertilization was evaluated 18 hours after insemination. Embryos were cultured in early cleavage medium before Day3 and in multiblast medium afterwards. All embryos were cultured in incubator at $37^{\circ} \mathrm{C}, 5 \% \mathrm{O}_{2}$ and $6 \% \mathrm{CO}_{2}$ concentration. Embryo development was evaluated on Day2, Day3 and Day5. Day2 or Day3 cleavage-stage embryos with at least three or six blastomeres respectively and with fragmentation $<20 \%$ according to Holte classification were eligible for cryopreservation. The criteria for good-quality embryo were: for Day 2 embryos, 4 to 5 cells with no fragmentation, for Day3 embryos, 7 to 9 cells with less than $20 \%$ fragmentation. 
In a FET cycle, patients were administered estrogen and progesterone sequentially for endometrial preparation before FET. Patients started with estrogen administered orally (Estradiol Valerate or Estradiol Femoston) $6 \mathrm{mg}$ per day with or without adding estrogen vaginally (Estradiol Femoston) 2 mg per day. Transvaginal ultrasonagraphy, serum E2, LH and progesterone level were measured at each visit weekly. Once the time of FET was determined, progesterone intramuscularly (60 mg per day) or Crinone (90 mg per day) vaginally was initiated daily. They both combined with dydrogesterone orally $30 \mathrm{mg}$ per day and estradiol orally $6 \mathrm{mg}$ per day. Intramuscular Progesterone or Vaginal Crinone was picked depending on patient preference. Patients who were undertaken Day 2 or Day 3 cleavage-staged embryo transferred started the progesterone 2 or 3 days before FET, respectively. The vitrification and thawing procedure were previously presented[11]. Embryo transfer was performed via the same flexible catheter under transabdominal ultrasound guidance. After FET, daily estrogen and progesterone administration continued until a negative pregnancy test at the 14th day after embryo transfer. If pregnancy was achieved, hormone administration continued until 12 weeks' gestation.

\section{Outcome measures and definitions}

In order to evaluate the impact of progesterone delivered regimens on clinical outcome, the primary outcome analyzed was live birth rate. Secondary outcomes included clinical pregnancy rate, newborn birthweight, large for gestational age (LGA), small for gestational age (SGA), preterm delivery rate. Live birth was defined as a delivery of a viable infant after the 28th gestational weeks. Clinical pregnancy is a pregnancy confirmed by the confirmation of gestational sac or heart beat. Gestational age was calculated from 14 days before the embryo transfer. Preterm birth was defined as delivery between 32 to 37 gestational weeks. SGA and LGA were defined as birthweight $<10$ th and $>90$ th percentile, respectively. Z score was administered to calculate birthweight adjusted for gestational age and newborn gender using the formula: $Z$ score $=\left(\chi^{-} \alpha\right) / \sigma$, where $\chi$ is the birthweight of the infant, $\alpha$ is the mean birthweight for the same sex and same gestational age in the reference group and $\sigma$ is the standard deviation of the reference group. The reference is the Chinese singletons newborns[12].

Statistical analysis

Patients and singletons live birth demographic baseline, cycle characteristics, clinical and neonatal outcomes were compared using Student's t-test, Wilcoxon rank sum tests, chi-square and Fisher's exact tests, as appropriate. Whether binary live birth and clinical pregnancy were modified by the regimen of progesterone was assessed by multivariable logistic regression adjusting for major covariates maternal age, $\mathrm{BMI}$, the route of estrogen administered, whether duration of estradiol treatment $>21$ days, whether at least one good quality embryo was transfered. Multivariable logistic regression was applied to evaluate the regimen of progesterone impact on neonatal outcomes adjusting for the major covariates mentioned above plus newborn gender. Adjusted odds ratios (OR) and $95 \%$ confidence intervals $(95 \% \mathrm{Cl})$ were reported. All analyses were conducted with spss statistics. $P$ value $<0.05$ was considered statistically significant. 


\section{Results}

This analysis included 856 women and 240 live birth singletons with the following outcomes: $44.04 \%$ clinical pregnancy rate, $35.63 \%$ live birth rate. There were 333 patients who were progesterone administered intramuscularly and 523 patients who were progesterone administered vaginally. Baseline demographics and characteristics were compared between patients with different progesterone regimens (Table 1). Among the 856 women, it did not reveal any significant difference for maternal age, BMI, whether there was at least one good quality embryo transferred, endometrium thickness at endometrium transform day, days of estrogen duration, E2, P, LH level at endometrium transform day between two groups. The proportion of patients with estradiol vaginally and orally delivered together in progesterone vaginally group was significantly higher than in progesterone intramuscularly group. Serum progesterone level at 14th day after embryo transfer was significantly higher in progesterone intramuscularly group than in progesterone vaginally group. No significant difference of live birth rate and clinical pregnancy rate was found between different progesterone regimens (Crude OR 1.181, 95Cl\% 0.895, 1.557, $\mathrm{p}=0.282$; Crude OR 1.170, 95\% Cl 0.879, 1.557, $\mathrm{p}=0.239$ )(Table 2). Controlling for maternal age, BMI, the route of estrogen administration, whether estradiol duration was longer than 21 days, whether there was at least one good quality embryo transferred, progesterone administered regimen did not modify the odds of achieving live birth (Adjusted OR 1.128, 95\% $\mathrm{Cl} 0.842,1.511, \mathrm{p}=0.420$ ) or clinical pregnancy (Adjusted OR $1.144,95 \% \mathrm{Cl} 0.863,1.518, \mathrm{p}=0.349$ ) (Table 2). Maternal age and at least one good quality embryo transferred were the only independent factors that increased the live birth rate and clinical pregnancy rate. 
Table 1

Baseline demographics and cycle characteristics according to different progesterone routes

\begin{tabular}{|c|c|c|c|}
\hline & $\begin{array}{l}\text { Progesterone } \\
\text { intramuscularly }(\mathrm{N}=333)\end{array}$ & $\begin{array}{l}\text { Crinone vaginally } \\
(N=523)\end{array}$ & $\mathbf{P}$ \\
\hline Maternal age (y) & $30.46 \pm 4.5$ & $31.0 \pm 4.5$ & 0.051 \\
\hline BMI & $21.47 \pm 3.1$ & $21.22 \pm 3.0$ & 0.26 \\
\hline At least one good quality embryo & 294 & 451 & 0.383 \\
\hline $\begin{array}{l}\text { Endometrium thickness at endometrium } \\
\text { transform day }(\mathrm{mm})\end{array}$ & $9.0(8.38,9.63)(\mathrm{N}=320)$ & $\begin{array}{l}8.9(8.4,9.4)(\mathrm{N}= \\
508)\end{array}$ & 0.355 \\
\hline \multicolumn{4}{|l|}{ Days of estradiol administration } \\
\hline$>21$ days & 24 & 36 & 0.856 \\
\hline$\leq 21$ days & 209 & 487 & \\
\hline \multicolumn{4}{|l|}{ Estrogen route } \\
\hline Orally and vaginally & 126 & 229 & 0.009 \\
\hline Orally only & 107 & 294 & \\
\hline $\begin{array}{l}\text { E2 level at endometrium transform day } \\
\text { (pmol/L) }\end{array}$ & $\begin{array}{l}1374(789.3,3843.3) \\
(\mathrm{N}=312)\end{array}$ & $\begin{array}{l}1342.5(822, \\
5291) \\
(\mathrm{N}=479)\end{array}$ & 0.340 \\
\hline $\begin{array}{l}\text { P level at endometrium transform day } \\
\text { (pmol/L) }\end{array}$ & $1.1(0.68,1.7)(\mathrm{N}=311)$ & $\begin{array}{l}1.2(0.65,1.85)(\mathrm{N} \\
=477)\end{array}$ & 0.663 \\
\hline $\begin{array}{l}\text { LH level at endometrium transform day } \\
\text { (pmol/L) }\end{array}$ & $8.8(4.78,14.11)(\mathrm{N}=311)$ & $\begin{array}{l}8.6(4.44,14.72) \\
(\mathrm{N}=477)\end{array}$ & 0.561 \\
\hline $\begin{array}{l}\text { P level at 14th day after embryo transfer } \\
\text { (pmol/L) }\end{array}$ & $\begin{array}{l}40.5(31.61,57.57)(\mathrm{N}= \\
262)\end{array}$ & $\begin{array}{l}14.95(8.29,25.55) \\
(N=410)\end{array}$ & $\begin{array}{l}< \\
0.001\end{array}$ \\
\hline
\end{tabular}


Table 2

Clinical outcomes according to different progesterone routes

\begin{tabular}{|c|c|c|c|c|c|c|}
\hline & $\begin{array}{l}\text { Progesterone } \\
\text { intramuscularly ( } N \\
=333)\end{array}$ & $\begin{array}{l}\text { Crinone } \\
\text { vaginally ( } N= \\
523)\end{array}$ & $\begin{array}{l}\text { Crude OR } \\
(95 \% \mathrm{Cl})\end{array}$ & $\mathbf{P}$ & $\begin{array}{l}\text { Adjusted OR } \\
(95 \% \mathrm{Cl})\end{array}$ & $P$ \\
\hline $\begin{array}{l}\text { Clinical } \\
\text { pregnancy }\end{array}$ & 155(46.55) & $222(42.45)$ & $\begin{array}{l}1.181 \\
(0.895 \\
1.557)\end{array}$ & 0.239 & $\begin{array}{l}1.144(0.863 \\
1.518) \text { a }\end{array}$ & 0.349 \\
\hline Live birth & 126(37.84) & $179(34.23)$ & $\begin{array}{l}1.170(0.879 \\
1.557)\end{array}$ & 0.282 & $\begin{array}{l}1.128(0.842, \\
1.511) \text { a }\end{array}$ & 0.420 \\
\hline
\end{tabular}

a: adjusted for maternal age, BMl, transfer with at least one good quality embryo, estrogen regimen, whether estrogen administration days $>21$. $\mathrm{Cl}=$ confidence interval; $\mathrm{OR}=$ odds ratio.

To further explore the progesterone regimen impact on birthweight and gestational age, a cohort of 240 live birth singletons from 856 patients was investigated. Neonatal outcomes stratified by the regimen of progesterone administered were presented in Table 3. Newborn gender, gestational age, mean birthweight, Z-scores, preterm delivery rate, SGA and LGA rate were not different across two groups (Table 3). In multivariate analyses (Table 3), the risk of preterm delivery (Adjusted OR 1.920,95\% Cl 0.603, 6.11, $\mathrm{p}=$ 0.269 ), the risk of LGA (Adjusted OR $0.862,95 \% \mathrm{Cl} 0.425,1.749, \mathrm{p}=0.681$ ), and SGA (Adjusted OR 0.227, $95 \% \mathrm{Cl} 0.027,1.934, \mathrm{p}=0.175$ ) were not significantly different between two groups after adjusting for maternal age, BMI, estradiol route, estrogen administration more than 21 days, transfer with at least one good quality embryo and newborn gender. 
Table 3

Neonatal outcomes of singletons live birth according to different progesterone routes

\begin{tabular}{|c|c|c|c|c|c|c|c|}
\hline $\begin{array}{l}\text { All } \\
\text { singletons }\end{array}$ & \multicolumn{3}{|c|}{$\begin{array}{l}\text { Progesterone intramuscularly } \\
(\mathrm{N}=95)\end{array}$} & \multicolumn{2}{|c|}{$\begin{array}{l}\text { Crinone } \\
\text { vaginally } \\
(N=145)\end{array}$} & \multicolumn{2}{|c|}{$\mathbf{P}$} \\
\hline \multicolumn{8}{|l|}{$\begin{array}{l}\text { Newborn } \\
\text { gender }\end{array}$} \\
\hline Female & \multicolumn{3}{|l|}{45} & \multicolumn{2}{|c|}{71} & \multicolumn{2}{|c|}{0.809} \\
\hline Male & \multicolumn{3}{|l|}{50} & \multicolumn{2}{|c|}{74} & & \\
\hline \multicolumn{8}{|l|}{$\begin{array}{l}\text { Gestational } \\
\text { Age }\end{array}$} \\
\hline $32-36$ & \multicolumn{3}{|l|}{7} & \multicolumn{2}{|c|}{7} & \multicolumn{2}{|l|}{0.411} \\
\hline$>37$ & \multicolumn{3}{|l|}{88} & \multicolumn{2}{|c|}{138} & & \\
\hline $\begin{array}{l}\text { Birth } \\
\text { weight }\end{array}$ & \multicolumn{3}{|l|}{$3349.19 \pm 487.2$} & \multicolumn{2}{|c|}{$\begin{array}{l}3365.58 \pm \\
469.5\end{array}$} & \multicolumn{2}{|c|}{0.744} \\
\hline \multirow[t]{2}{*}{ Z score } & \multicolumn{3}{|l|}{$0.357 \pm 1.047$} & \multicolumn{2}{|c|}{$0.345 \pm 1.023$} & \multicolumn{2}{|c|}{0.928} \\
\hline & $\begin{array}{l}\text { Progesterone } \\
\text { intramuscularly } \\
(\mathrm{N}=95)\end{array}$ & $\begin{array}{l}\text { Crinone } \\
\text { vaginally } \\
(N=145)\end{array}$ & $\begin{array}{l}\text { Crude } \\
\text { OR( }(95 \% \mathrm{Cl})\end{array}$ & $P$ & $\begin{array}{l}\text { Adjusted } \\
\text { OR( }(95 \% \mathrm{Cl})\end{array}$ & & $P$ \\
\hline $\begin{array}{l}\text { Preterm } \\
\text { delivery }\end{array}$ & 7 & 7 & $\begin{array}{l}1.568(0.532, \\
4.623)\end{array}$ & 0.411 & $\begin{array}{l}1.920(0.603, \\
6.110) \mathrm{a}\end{array}$ & & 0.269 \\
\hline SGA & 1 & 6 & $\begin{array}{l}0.246(0.029 \\
2.080)\end{array}$ & 0.319 & $\begin{array}{l}0.227(0.027, \\
1.934) \mathrm{a}\end{array}$ & & 0.175 \\
\hline LGA & 14 & 27 & $\begin{array}{l}0.819(0.410, \\
1.637)\end{array}$ & 0.572 & $\begin{array}{l}0.862(0.425, \\
1.749) \mathrm{a}\end{array}$ & & 0.681 \\
\hline
\end{tabular}

Data are presented as mean $\pm S D$ for continuous variables in formal distribution. $P$ values were assessed with the use of $t$ tests or Wilcoxon rank sum tests or chi-square (Fisher's exact tests as appropriate). a: adjusted for maternal age, BMI, transfer with at least one good quality embryo, estrogen regimen, whether estrogen administration days $>21$, newborn gender. SGA stands for small for gestational age. LGA stands for large for gestational age.

In order to investigate the circulating serum progesterone impact on newborn gestational weeks and birth weight, a cohort of 262 patients with progesterone intramuscularly and 77 live birth singletons was further investigated. We did not analysis vaginal progesterone cohort because systemic serum progesterone value was not the reflection of progesterone Crinone vaginally absorbed[13]. From the cohort, clinical and neonatal outcomes of patients with serum progesterone level $>41.82 \mathrm{pmol} / \mathrm{L}$ and $\leq$ $41.82 \mathrm{pmol} / \mathrm{L}$ at the 14th day after embryo transfer were presented in Table $4.41 .82 \mathrm{pmol} / \mathrm{L}$ was the median value of serum progesterone level at the Hcg test day for patients with progesterone 
intramuscularly administered. Patients with serum $\mathrm{P}$ level $>41.82 \mathrm{pmol} / \mathrm{L}$ demonstrated higher clinical pregnancy rate and live birth rate than patients with serum $P$ level $\leq 41.82 \mathrm{pmolL}$ both in univariate analysis and multivariate analysis adjusting for maternal age, BMI, whether at least one good quality embryo was transfered, estrogen regimen, whether estrogen administration days $>21$ (Adjusted OR 1.670, $95 \% \mathrm{Cl} 1.005,2.774, \mathrm{p}=0.048$; Adjusted OR 1.690, 95\% Cl 1.002, 2.849, $\mathrm{p}=0.049$ ). While birthweight, Zscore, preterm delivery rate, LGA and SGA rate were not different between these two groups. Multivariate analysis was not performed for preterm delivery rate, LGA and SGA rate because the number in this category was too small.

Table 4

Reproductive and neonatal outcomes between $\mathrm{P}$ level $>41.82 \mathrm{pmol} / \mathrm{L}$ and $\mathrm{P}$ level $\leq 41.82 \mathrm{pmol} / \mathrm{L}$ groups when progesterone intramuscularly was used in HRT-FET cycles

\begin{tabular}{|c|c|c|c|c|c|c|}
\hline & $\begin{array}{l}P \text { level > } \\
41.82 \mathrm{pmol} / \mathrm{L} \\
(\mathrm{N}=131)\end{array}$ & $\begin{array}{l}\mathrm{P} \text { level } \leq \\
41.82 \mathrm{pmol} / \mathrm{L} \\
(\mathrm{N}=131)\end{array}$ & $\begin{array}{l}\text { Crude OR } \\
(95 \% \mathrm{Cl})\end{array}$ & $P$ & $\begin{array}{l}\text { Adjusted OR } \\
(95 \% \mathrm{Cl})\end{array}$ & $\mathbf{P}$ \\
\hline $\begin{array}{l}\text { Clinical } \\
\text { Pregnancy }\end{array}$ & $72(54.9)$ & $55(41.98)$ & $\begin{array}{l}1.686(1.034, \\
2.940)\end{array}$ & 0.036 & $\begin{array}{l}1.670(1.005 \\
2.774) \mathrm{a}\end{array}$ & 0.048 \\
\hline Live Birth & $59(45.04)$ & $43(32.82)$ & $\begin{array}{l}1.677(1.016, \\
2.769)\end{array}$ & 0.043 & $\begin{array}{l}1.690(1.002 \\
2.849) a\end{array}$ & 0.049 \\
\hline $\begin{array}{l}\text { Singletons } \\
\text { Neonatal } \\
\text { outcome }\end{array}$ & $\begin{array}{l}\text { P level > } \\
41.82 \mathrm{pmol} / \mathrm{L} \\
(\mathrm{N}=42)\end{array}$ & $\begin{array}{l}\text { P level } \leq \\
41.82 \mathrm{pmol} / \mathrm{L} \\
(\mathrm{N}=35)\end{array}$ & $\begin{array}{l}\text { Crude OR } \\
(95 \% \mathrm{Cl})\end{array}$ & $P$ & & \\
\hline Birthweight & $\begin{array}{l}3331.79 \pm \\
421.19\end{array}$ & $\begin{array}{l}3354.03 \pm \\
643.51\end{array}$ & \multicolumn{4}{|c|}{0.861} \\
\hline Z score & $\begin{array}{l}0.2778 \pm \\
0.9658\end{array}$ & $\begin{array}{l}0.6248 \pm \\
1.1147\end{array}$ & \multicolumn{4}{|c|}{0.153} \\
\hline $\begin{array}{l}\text { Preterm } \\
\text { delivery }\end{array}$ & 2 & 5 & $\begin{array}{l}0.391(0.074, \\
2.051)\end{array}$ & \multicolumn{3}{|c|}{0.444} \\
\hline LGA & 5 & 8 & $\begin{array}{l}0.610(0.194, \\
1.917)\end{array}$ & \multicolumn{3}{|c|}{0.393} \\
\hline SGA & 1 & 0 & / & \multicolumn{3}{|l|}{ / } \\
\hline
\end{tabular}

\section{Discussion}

FET cycle number has increased significantly recent years due to single embryo transfer and freeze all policy. Though no best endometrial preparation protocol for FET exists, hormone replacement treatment (HRT) is preferred by many centers due to the controlled exposure to exogenous hormones and 
adjustment of the timing of transfer. In HRT-FET cycles, estradiol and progesterone are sequentially administered to synchronize the window of embryo implantation with embryo transfer. Once the adequate proliferation of the endometrium is achieved, progesterone daily starts before scheduled embryo transfer, which induces appropriate progesterone receptors and develops endometrial receptivity. However, the ideal administration route, duration and the dosage of progesterone have not been well defined. Vaginal Crinone and intramuscular progesterone are two common luteal support regimens. Vaginally delivered progesterone showed advantage with less side effect and better compliance. It reached the uterus directly and induced higher progesterone concentration in the uterine endometrium with relatively lower circulating $P$ level compared to intramuscular regimen[14]. Vaginal administration also ensured a stable concentration in the endometrium when serum P level was low. Report has suggested vaginal progesterone administration helped decrease uterine contraction frequency and lowered the risk of displacement of embryo in fresh IVF-ET cycles[15].

Since the higher risk of LGA and post-date newborns from FET singletons compared to fresh IVF-ET cycles was reported[6]. Several studies reported the negative impact of very high progesterone level on endometrium maturation and implantation in non-human studies[16, 17]. Serum progesterone level has been tightly associated with endometrium angiogenesis and placenta formation which influence newborn birthweight and gestational weeks[9]. Hence we want to explore whether large-spanned circulation progesterone values by different luteal support regimens make a difference in live birth rate, newborn gestational weeks and birthweight.

From our study, we showed that large spanned serum $\mathrm{P}$ level induced by different progesterone regimen did not impact live birth rate in HRT-FET cycles. Similar results showed that progesterone vaginally administered resulted comparable results with intramuscular regimen as luteal support in fresh IVF cycles [18-20]. We further analyzed neonatal outcomes stratified by different progesterone regimen and found no significant difference of infant birthweight, preterm delivery rate, SGA and LGA rate. The reason why we didn't see the higher peripheral serum $P$ by intramuscularly delivered progesterone induced higher birthweight and longer gestational weeks in our cohort may be speculated that the placenta formation and angiogenesis could be influenced by not only circulating progesterone, but also uterine local progesterone level. Vaginally delivered progesterone might create the high concentration of progesterone in uterus locally but it is hard to detect. In our cohort patients received dydrogesterone orally at the same time in case of vaginal malabsorption, though dydrogesterone orally taken did not contribute to the serum progesterone value. Additionally, it shouldn't be neglected that vaginal progesterone uptake distribution and metabolism vary tremendously between patients.

Some studies showed low serum progesterone on the day of embryo transfer is associated with inferior clinical pregnancy both in artificial FET and fresh IVF-ET cycles[21-23]. It is difficult to compare findings from studies that examine P concentration at different stages of cycle. In our study we analyzed the circulating progesterone concentration of 14th day after embryo transfer, which is also Hcg test day. Almost no endogenous progesterone from placenta is present at this time. Only little progesterone from trophoblasts contributes to serum progesterone concentration in the peri-implantation period. Our results 
showed higher serum $\mathrm{P}$ level was associated with higher live birth rate and clinical pregnancy rate in progesterone intramuscularly cohort. Serum absolute progesterone level positively impacted the successful rate in artificial FET cycles when intramuscularly delivered progesterone was applied as luteal support. Clinicians could optimize intramuscular progesterone supplementation according to the $P$ monitoring. Our findings confirmed previous data that serum higher progesterone level during mid-luteal phase got higher successful rate than the lower P level counterparts in HRT-FET cycles. Luteal progesterone level outside the range limits reduced clinical pregnancy rate[24-26]. We further explored newborn birthweight and gestational age in intramuscular progesterone cohort. Our study revealed that systemic $P$ level higher than $41.82 \mathrm{pmol} / \mathrm{L}$ did not increase birthweight and prolong gestational weeks, but it increased live birth rate. Thus it is advised to monitor systemic $\mathrm{P}$ concentration and provide higher progesterone dose as needed in intramuscular progesterone cohort for optimizing live birth rate without impact on neonatal outcomes. We did not analyze the circulating progesterone value impact in vaginal progesterone cohort because systemic progesterone concentration does not serve as a surrogate marker for efficacy of vaginal progesterone[13].

The present study has following strengths. The analysis was performed in single IVF center which guaranteed the same laboratory procedures and sonographers. The analysis only included patients with their first FET cycles to assure the relative good quality embryo transfer and exclude the recurrent embryo failure cases. We excluded blastocyst transfer to alleviate prolonged in vitro embryo culture impact on neonatal outcomes[27, 28]. To control for infant gender and gestational age bias, $z$ score was calculated across different groups. There were limitations in this study. It is a retrospective analysis and selection bias was possible. Although we accounted for some associated factors in multivariate analysis, unknown factors might have affected the results. Large randomized prospective study is needed. We couldn't follow up the patient about obstetrics details, which impeded us to assess perinatal outcomes like gestational hypertension, pre-eclampsia, placenta accrete and previa. The perinatal details would allow us a better understanding of high serum progesterone level's impact on placental formation in late pregnancy.

\section{Conclusion}

This study showed progesterone vaginally or intramuscularly didn't impact live birth rate and neonatal outcomes in artificial FET cycles. Relatively higher serum progesterone level induced by intramuscular regimen did not increase newborn birthweight or prolong gestational weeks compared to vaginal regimen. Intramuscular progesterone supplementation during HRT-FET cycles was associated with improved live birth rate when progesterone concentration at day 14 post-FET was higher than $41.82 \mathrm{pmol} / \mathrm{L}$. Monitoring serum progesterone level and optimizing progesterone dose as needed in intramuscular progesterone HRT-FET cycles has a role in improving clinical outcomes without impact on neonatal outcomes.

\section{Abbreviations}

HRT 
hormone replacement therapy

FET

frozen-thawed embryo transfer

cos

controlled ovarian stimulation

IVF

in vitro fertilization

ICSI

intracytoplasmic sperm injection

LGA

large for gestational age

SGA

small for gestational age

$95 \% \mathrm{Cl}$

95\% confidence intervals

OR

odds ratios

BMI

body mass index

\section{Declarations}

Ethics approval and consent to participate: Institutional review board and ethics committee of Shanghai General Hospital approval was obtained.

Consent for publication: Not applicable.

Availability of data materials: The datasets used during the current study are available from the corresponding author on reasonable request.

Competing interests: The authors declare that they have no competing interests. Funding: There is no funding for this work.

Authors contribution: Y.L. and Y.W. were involved in study concept and design. Y.L. collected and analyzed the data. Y.L drafted the article. Y.W. revised it for important intellectual content. All authors have approved the submitted version and agreed both to be personally accountable for the author's own contributions and to ensure that questions related to the accuracy or integrity of any part of the work.

\section{References}

1. Wennerholm UB, Henningsen AK, Romundstad LB, Bergh C, Pinborg A, Skjaerven R, Forman J, Gissler M, Nygren KG, Tiitinen A: Perinatal outcomes of children born after frozen-thawed embryo transfer: a 
Nordic cohort study from the CoNARTaS group. Hum Reprod 2013, 28:2545-2553.

2. Shi Y, Sun Y, Hao C, Zhang H, Wei D, Zhang Y, Zhu Y, Deng X, Qi X, Li H, et al: Transfer of Fresh versus Frozen Embryos in Ovulatory Women. N Engl J Med 2018, 378:126-136.

3. Sazonova A, Kallen K, Thurin-Kjellberg A, Wennerholm UB, Bergh C: Obstetric outcome in singletons after in vitro fertilization with cryopreserved/thawed embryos. Hum Reprod 2012, 27:1343-1350.

4. Opdahl S, Henningsen AA, Tiitinen A, Bergh C, Pinborg A, Romundstad PR, Wennerholm UB, Gissler M, Skjaerven R, Romundstad LB: Risk of hypertensive disorders in pregnancies following assisted reproductive technology: a cohort study from the CoNARTaS group. Hum Reprod 2015, 30:17241731.

5. Maheshwari A, Pandey S, Amalraj Raja E, Shetty A, Hamilton M, Bhattacharya S: Is frozen embryo transfer better for mothers and babies? Can cumulative meta-analysis provide a definitive answer? Hum Reprod Update 2018, 24:35-58.

6. Pinborg A, Henningsen AA, Loft A, Malchau SS, Forman J, Andersen AN: Large baby syndrome in singletons born after frozen embryo transfer (FET): is it due to maternal factors or the cryotechnique? Hum Reprod 2014, 29:618-627.

7. Pereira N, Elias RT, Christos PJ, Petrini AC, Hancock K, Lekovich JP, Rosenwaks Z: Supraphysiologic estradiol is an independent predictor of low birth weight in full-term singletons born after fresh embryo transfer. Hum Reprod 2017, 32:1410-1417.

8. Zhang W, Ma Y, Xiong Y, Xiao X, Chen S, Wang X: Supraphysiological serum oestradiol negatively affects birthweight in cryopreserved embryo transfers: a retrospective cohort study. Reprod Biomed Online 2019, 39:312-320.

9. Walter LM, Rogers PA, Girling JE: The role of progesterone in endometrial angiogenesis in pregnant and ovariectomised mice. Reproduction 2005, 129:765-777.

10. Pirtea P, de Ziegler D, Ayoubi JM: Implantation rates of euploid embryos are not influenced by the duration of estradiol priming, but the hormonal environment-estradiol and progesterone-may affect placentation. Fertil Steril 2019, 111:1117-1118.

11. Zhang J, Liu H, Mao X, Chen Q, Si J, Fan Y, Xiao Y, Wang Y, Kuang Y: Effect of endometrial thickness on birthweight in frozen embryo transfer cycles: an analysis including 6181 singleton newborns. Hum Reprod 2019, 34:1707-1715.

12. Dai L, Deng C, Li Y, Zhu J, Mu Y, Deng Y, Mao M, Wang Y, Li Q, Ma S, et al: Birth weight reference percentiles for Chinese. PLOS One 2014, 9:e104779.

13. Boelig RC, Zuppa AF, Kraft WK, Caritis S: Pharmacokinetics of vaginal progesterone in pregnancy. Am J Obstet Gynecol 2019, 221:263.e261-263.e267.

14. Cicinelli E, Schonauer LM, Galantino P, Matteo MG, Cassetta R, Pinto V: Mechanisms of uterine specificity of vaginal progesterone. Hum Reprod 2000, 15 Suppl 1:159-165.

15. Fanchin R, Righini C, de Ziegler D, Olivennes F, Ledee N, Frydman R: Effects of vaginal progesterone administration on uterine contractility at the time of embryo transfer. Fertil Steril 2001, 75:1136-1140. 
16. Lonergan P: Influence of progesterone on oocyte quality and embryo development in cows. Theriogenology 2011, 76:1594-1601.

17. Nogueira MF, Melo DS, Carvalho LM, Fuck EJ, Trinca LA, Barros CM: Do high progesterone concentrations decrease pregnancy rates in embryo recipients synchronized with PGF2alpha and eCG? Theriogenology 2004, 61:1283-1290.

18. Mitwally MF, Diamond MP, Abuzeid M: Vaginal micronized progesterone versus intramuscular progesterone for luteal support in women undergoing in vitro fertilization-embryo transfer. Fertil Steril 2010, 93:554-569.

19. Zarei A, Sohail P, Parsanezhad ME, Alborzi S, Samsami A, Azizi M: Comparison of four protocols for luteal phase support in frozen-thawed Embryo transfer cycles: a randomized clinical trial. Arch Gynecol Obstet 2017, 295:239-246.

20. Shapiro DB, Pappadakis JA, Ellsworth NM, Hait HI, Nagy ZP: Progesterone replacement with vaginal gel versus i.m. injection: cycle and pregnancy outcomes in IVF patients receiving vitrified blastocysts. Hum Reprod 2014, 29:1706-1711.

21. Labarta E, Mariani G, Holtmann N, Celada P, Remohi J, Bosch E: Low serum progesterone on the day of embryo transfer is associated with a diminished ongoing pregnancy rate in oocyte donation cycles after artificial endometrial preparation: a prospective study. Hum Reprod 2017, 32:2437-2442.

22. Boynukalin FK, Gultomruk M, Turgut E, Demir B, Findikli N, Serdarogullari M, Coban O, Yarkiner Z, Bahceci M: Measuring the serum progesterone level on the day of transfer can be an additional tool to maximize ongoing pregnancies in single euploid frozen blastocyst transfers. Reprod Biol Endocrinol 2019, 17:102.

23. Cedrin-Durnerin I, Isnard T, Mahdjoub S, Sonigo C, Seroka A, Comtet M, Herbemont C, Sifer C, Grynberg M: Serum progesterone concentration and live birth rate in frozen-thawed embryo transfers with hormonally prepared endometrium. Reprod Biomed Online 2019, 38:472-480.

24. Yovich JL, Conceicao JL, Stanger JD, Hinchliffe PM, Keane KN: Mid-luteal serum progesterone concentrations govern implantation rates for cryopreserved embryo transfers conducted under hormone replacement. Reprod Biomed Online 2015, 31:180-191.

25. Basnayake SK, Volovsky M, Rombauts L, Osianlis T, Vollenhoven B, Healey M: Progesterone concentrations and dosage with frozen embryo transfers - What's best? Aust NZ J Obstet Gynaecol 2018, 58:533-538.

26. Alsbjerg B, Thomsen L, Elbaek HO, Laursen R, Povlsen BB, Haahr T, Humaidan P: Progesterone levels on pregnancy test day after hormone replacement therapy-cryopreserved embryo transfer cycles and related reproductive outcomes. Reprod Biomed Online 2018, 37:641-647.

27. Zhang J, Wang Y, Liu H, Mao X, Chen Q, Fan Y, Xiao Y, Kuang Y: Effect of in vitro culture period on birth weight after vitrified-warmed transfer cycles: analysis of 4,201 singleton newborns. Fertil Steril 2019, 111:97-104.

28. Ginstrom Ernstad E, Spangmose AL, Opdahl S, Henningsen AA, Romundstad LB, Tiitinen A, Gissler M, Wennerholm UB, Pinborg A, Bergh C, Malchau SS: Perinatal and maternal outcome after vitrification 
of blastocysts: a Nordic study in singletons from the CoNARTaS group. Hum Reprod 2019, 34:22822289. 